\title{
Impact of post-growth thermal annealing on emission of InGaN/GaN multiple quantum wells
}

\author{
Yung-Chen Cheng ${ }^{1}$, S. Juršènas ${ }^{*}, 2$, Shih-Wei Feng ${ }^{1}$, C. C. Yang ${ }^{1}$, Cheng-Ta Kuo ${ }^{3}$, \\ and Jian-Shihn Tsang ${ }^{3}$ \\ ${ }^{1}$ Graduate Institute of Electro-Optical Engineering and Department of Electrical Engineering, \\ National Taiwan University 1, Roosevelt Road, Sec. 4, Taipei, Taiwan, R.O.C. \\ 2 Institute of Materials Science and Applied research, Vilnius University Sauletekio al. 9-III, \\ LT-2040 Vilnius, Lithuania \\ 3 Advanced Epitaxy Technology Inc., Hsinchu Industrial Park, Hsinchu, Taiwan, R.O.C.
}

Received 15 September 2003, accepted 17 September 2003

Published online 29 December 2003

PACS 78.47.+p, 78.55.Cr, 78.67.De

Photoluminescence, photoluminescence excitation and time-resolved luminescence study of three $\mathrm{In}_{0.15} \mathrm{Ga}_{0.85} \mathrm{~N} / \mathrm{GaN}$ multiple quantum wells (MQWs) with well-widths of 2, 3, 4-nm, upon thermal annealing at $800{ }^{\circ} \mathrm{C}$ for $30 \mathrm{~min}$ is presented. Blueshift of luminescence and pronounced changes in the absorbance indicate on remarkable interdiffusion of indium at the quantum well barrier interface for thin MQWs. While pronounced redshift in luminescence and increase in the excitation lifetime is attributed to prevalence of strain-induced field-effect for the thicker MQWs. Site-selective spectroscopy and microstructure analysis monitors improvement of the MQW interface quality of the thicker layers as well as reduction of disorder of in the quantum well layer upon post-growth thermal annealing.

(C) 2004 WILEY-VCH Verlag GmbH \& Co. KGaA, Weinheim

1 Introduction InGaN-based multiple quantum wells (MQWs) are the key structures for production of high efficiency, long lifetime violet, blue, and green light emitting diodes and laser diodes [1, 2]. During the epitaxial growth of InGaN/GaN structures and during the fabrication of devices, active layers undergo several high-temperature treatments [1]. After these thermal treatments, the distribution of the indium composition and the strain in quantum wells may be changed due to both ordinary diffusion [3-5] and "up-hill", strain induced, diffusion [6-9] effects.

Usually thermal annealing, is related to indium and gallium interdiffusion across the interface of a quantum-well and a barrier [3-5]. This leads to corresponding blueshift in photoluminescence (PL) and absorbance spectra [5]. A complete disappearance of InGaN/GaN MQWs after annealing at high temperature has been observed [4]. On the other hand the interdiffusion of indium and gallium in InGaN alloys is complicated by the immiscibility of InN and GaN, which can result in phase separation in InGaN layers [6, 7]. Since InGaN alloys usually are grown at temperatures below $1473 \mathrm{~K}$, the critical temperature above which InN-GaN system is completely miscible [10,11], phase separation is expected in all InGaN alloys based on thermodynamics considerations. The phenomenon of InGaN phase separation [6-14] are subjects of considerable interest, since it has been reported that InN-rich regions play a significant role in the mechanism of emission of nitride-based light emitting diodes and laser structures [15-19]. Phase separation has been observed using several growth techniques, preferably in the films of larger thickness $[6-9,12]$. The observed phase separation is evidently driven by strain due to the mixing of the two lattice mismatched components of the InGaN alloy system. Indium atoms are excluded from the InGaN lattice to form an alloy of different composition and reduce the strain energy of the system. It

" Corresponding author: e-mail: saulius.jursenas@ff.vu.It, Phone: + 37052 366027, Fax: +3705 2366059 
is predicted that for standard growing temperature spinodal decomposition is expected for indium concentration larger than $20 \%[10,11]$. It has been reported, that upon post-growth thermal annealing, indium rich regions are formed in InGaN quantum wells $[6-9,13]$. On the other hand, thermal annealing can lead to pronounced redistribution of the strain energy and reduce the phase separation, which is very sensitive to the strain effects. Both phase separation and strain effects are expected to enhance with increasing of the InGaN well width. Thus variation of indium compositional disorder and strain in asgrown samples of different well width can lead to changes in the dominating In separation mechanism upon thermal annealing.

Here we report on photoluminescence excitation (PLE) approaches combined with site-selective and time-resolved photoluminescence (PL) studies of the effect of post-growth thermal treatment on InGaN/GaN MQWs of different well thickness. We show that InGaN/GaN interface has a major impact on post-growth thermal-annealing related changes in the optical properties and material quality of InGaN/GaN MQWs of various thickness.

2 Experimental The samples were grown on c-plane sapphire by metal organic chemical vapor deposition. Following the deposition of $30 \mathrm{~nm} \mathrm{GaN}$ buffer layer and a $2.3 \mu \mathrm{m} \mathrm{GaN}$ layer, QW structures consisting of five pairs of $\mathrm{In}_{0.15} \mathrm{Ga}_{0.85} \mathrm{~N}$ well and $10 \mathrm{~nm}$-thick GaN barrier were grown. Three samples of different well widths at 2,3, and $4 \mathrm{~nm}$ were prepared and referred to as samples w20, w30 and w40, respectively. The growth temperatures were 1010 and $720{ }^{\circ} \mathrm{C}$ for $\mathrm{GaN}$ and $\mathrm{InGaN}$, respectively. Asgrown samples were thermally annealed in a quartz tube furnace at $800^{\circ} \mathrm{C}$ in nitrogen ambient for $30 \mathrm{~min}$.

The samples were placed in a cryostat for the temperature-dependent measurements. PLE experiments were conducted using quasi-monochromatic excitation light source from a xenon lamp dispersed by a 0.15-m monochromator. A Verdi-pumped mode-locked Ti:sapphire laser with $100 \mathrm{fs}$ pulse width and $76 \mathrm{MHz}$ in pulse repetition rate was frequency doubled by a BBO crystal. The excitation pulses we used were at $3.177 \mathrm{eV}$. The luminescence from the sample was collected and focused into a spectrometer before imaging in a Hamamatsu streak camera. The temporal resolution of operation mode was 4.74 ps.

3 Results and discussion Figure 1 shows normalized PLE spectra, taken at the peak position, and normalized PL spectra of as-grown a) and annealed b) samples at $10 \mathrm{~K}$. Main luminescence spectral properties are typical for low temperature emission of the localized exciton states in InGaN/GaN MQWs $[16,18,20]$. PLE spectra of as-grown w20 sample shows typical dependencies of a MQW structure, reflecting variation in a two dimensional density of states of $\mathrm{QW}(\mathrm{InGaN})$ and barrier $(\mathrm{GaN})$ materials. Peaks at $3.103 \mathrm{eV}$ and $3.503 \mathrm{eV}$ can be related to the exciton positions in InGaN and GaN layers, correspondingly.

By the increase of the well-width, a pronounced changes in the region of $\mathrm{GaN}$ barrier layers can be distinguished. Instead of expected step-like dependencies, as it is seen for w20 structure, an abrupt dependence on the excitation photon energy for $h v>3.50 \mathrm{eV}$ is observed. Changes of luminescence signal upon increase in the photon energy above the bandgap of the material can be attributed to the enhancement of nonradiative capture of the excited species with higher excess energy. In order to produce luminescence from QW states, excitons created by photoexcitation of the GaN barrier layer have to be captured by a well, thus to overcome the InGaN-GaN interface. Excitations with larger excess energy migrates for larger distances, thus have larger probability to be captured by nonradiative capture centers. Thus, narrowing of the PLE spectra are related to the density of the nonradiative capture centers, most of that are concentrated at the interfaces. As-grown samples show significant increase in the number of nonradiative centers, related to the GaN barrier with the increase in the well-width. This was also prooved by site-selective PL experiments (not shown).

Differences in degree of disorder and strain of as grown samples of various well-width leads to different impact of thermal annealing on luminescence of localized states. Luminescence band of w20 MQWs blueshifts (about $20 \mathrm{meV}$ ) together with the absorbance-edge upon thermal annealing. This might be related to indium interdiffusion to the barrier region [3-5]. In contrast, luminescence band of w30 sam- 

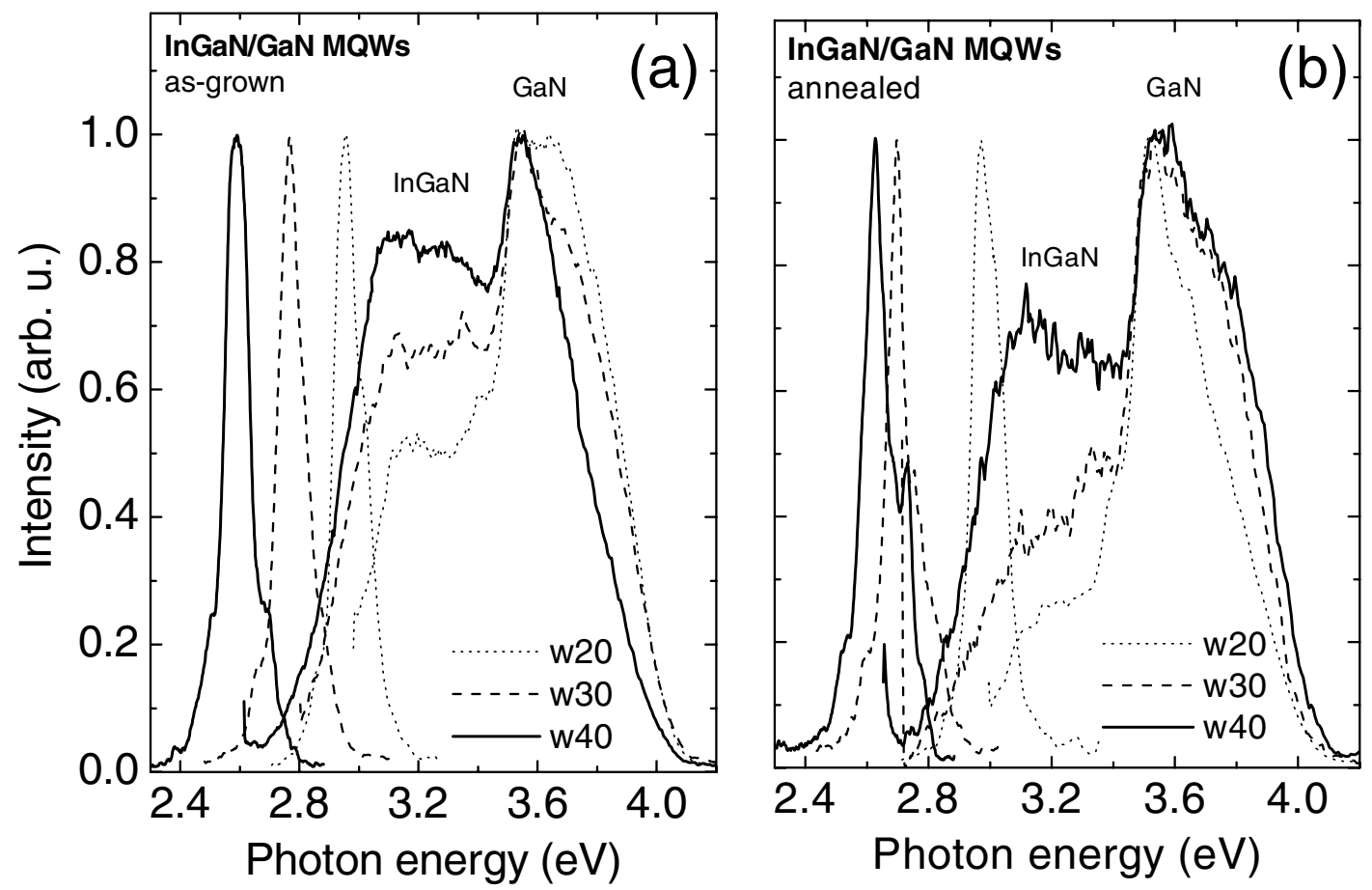

Fig. 1 Normalized at $3.50 \mathrm{eV}$ PLE spectra of as-grown a) and annealed b) InGaN/GaN MQWs of various well width. Normalized PL spectra are shown for comparison on the left-hand side.

ple redshifts $(71 \mathrm{meV})$ and that of w40 blueshifts $(45 \mathrm{meV})$ while absorbance edge show little changes in both later cases. Such behavior might be affected by several impacts such as, indium diffusion, increase in disorder due to indium decomposition, strain redistribution and strain related spinodal decomposition.

The PLE spectral changes are most pronounced for the GaN barrier region. PLE spectra show rapid decrease in the PL signal for excitation photon energy larger than GaN bandgap. Thus InGaN-GaN interface related nonradiative recombination is significantly enhanced upon thermal annealing. Barrier layer related PLE spectral changes diminishes with increase of the well-width. Even improvement material quality is observed for w40 MQWs upon thermal annealing. As-grown and annealed samples show opposite trends in InGaN-GaN interface related defect concentration by increasing of well-width: as-grown structures shows the best quality for the thinnest QWs and InGaN-GaN interface quality decreases with the well-width, while annealed samples show the worse quality of the thinnest MQWs and improvement of the quality by increase of the well-width.

Variation of exciton lifetime at low temperatures with the well-width indicates on importance of the is built-in field effect [21-23]. The low temperature lifetime of as-grown samples increases from $1.8 \mathrm{~ns}$ to $9 \mathrm{~ns}$ and $137 \mathrm{~ns}$ for w20, w30 and w40 samples, respectively. Upon thermal annealing of the w20 sample low temperature lifetime is almost the same, what is in disagreement with the decrease in emission efficiency. However, capture process of delocalized excitons to nonradiative centers at the interface can be very fast $(<1 \mathrm{ps})$ and probably cannot be detected because of low time-resolution of our system. After the excitons are localized they show the same lifetime. Thicker w30 sample shows increase in the lifetime from $9 \mathrm{~ns}$ to $41 \mathrm{~ns}$ upon thermal annealing. This is in line with the luminescence redshift and the same dynamic in the luminescence efficiency. Such trends might be accounted predominantly for the increase of the built-in field effect.

Summarizing, thinner samples, that initially are of the best optical quality, are almost destroyed by indium interdiffusion into barrier layer upon thermal annealing. Annealing leads to changes in indium content as it is seen by blueshift of the InGaN related absorption and formation of large amount of non- 
radiative defects at the interfaces. This is also monitored by microscopy images [24]. As consequence luminescence efficiency is significantly reduced. While the thicker samples are already very disordered due to indium segregation on growing surface. This leads to increase in the number of surface related defects in as grown samples. Annealing improves material quality, however for w30 sample annealing leads to formation more strain affected structure, what leads to decrease in efficiency due to built-in field effect. Phase separation in any alloy requires long range diffusion and thus correlation should exist between phase separation and period of the time required for the growth of the film. Therefore strain associated with thin InGaN layers could also stabilize the alloys against phase separation. For the thickest samples thermal annealing indeed improves material quality and leads to better luminescence efficiency. This imply that indium preferentially reorganized in the InGaN layer rather than diffused into GaN barriers. However as grown sample already possesses many defects on the growing surfaces of MQWs. This, together with increased built field effect, makes efficiency worse than that of the smaller thickness sample.

Acknowledgements This research was partially supported by the joint Lithuanian-Latvian-Taiwan grant and by National Science Council, The Republic of China, under the grants of NSC 90-2112-M-002-052, NSC 90-2215-E002-027, and NSC 90-2215-E-002-041.

\section{References}

[1] S. Nakamura and G. Fasol, The Blue Laser Diode: GaN Based Light Emitters and Lasers (Springer, Berlin, 1997).

[2] A. Žukauskas, M. S. Shur, and R. Gaska, Introduction to Solid-State Lighting (Wiley, New York, 2002).

[3] J.-S. Tsang, J.-D. Guo, S.-H. Chan, M.-S. Feng, and C.-Y. Chang, Jpn. J. Appl. Phys. 36, 1728 (1997).

[4] M. D. McCluskey, L. T. Romano, B. S. Krusor, N. M. Jonson, T. Suski, and J. Jun, Appl. Phys. Lett. 73, 1281 (1998).

[5] C.-C. Chou, C.-M. Lee, and J.-I. Chyj, Appl. Phys. Lett. 78, 314 (2001).

[6] R. Singh, D. Doppalapudi, T. D. Moustakas, and L. T. Romano, Appl. Phys. Lett. 70, 1089 (1997).

[7] M. D. McCluskey, L. T. Romano, B. S. Krusor, D. P. Bour, N. M. Johnson, and S. Brennan, Appl. Phys. Lett. 72, 1730 (1998)

[8] D. Doppalapudi, S. N. Basu, K. F. Ludvig, Jr., and T. D. Moustakas, J. Appl. Phys. 84, 1389 (1998).

[9] Y.-T. Moon, D.-J. Kim, K.-M. Song, C.-J. Coi, S.-H. Han, T.-Y. Seong, and S.-J. Park, J. Appl. Phys. 89, 6514 (2001).

[10] I. H. Ho and G. B. Stringfellow, Appl. Phys. Lett. 69, 2701 (1996).

[11] S. Yu. Karpov, J. Nitride Semicond. Res. 3, 16 (1998).

[12] K. Osamura, K. Nakajima, and Y. Murakami, Solid State Commun. 11, 617 (1972).

[13] Y.-S. Lin, K.-J. Ma, C. Hsu, Y.-Y. Chung, C.-W. Liu, S.-W. Feng, Y.-C. Cheng, M.-H. Mao, C. C. Yang, H.-W. Chuang, C.-T. Kuo, J.-S. Tsang, and T. E. Weirich, Appl. Phys. Lett. 80, 2571 (2002).

[14] S.-W. Feng, Y.-Y. Chung, C.-W. Liu, Y.-C. Cheng, C. C. Yang, M.-H. Mao, Y.-S. Lin, K.-J. Ma, and J.-I. Chyi, Appl. Phys. Lett. 80, 4375 (2002).

[15] S. Chichibu, T. Azuhata, T. Sota, and S. Nakamura, Appl. Phys. Lett. 70, 2822 (1997).

[16] S. Chichibu, T. Sota, K. Wada, and S. Nakamura, J. Vac. Sci. Technol. B 16, 2204 (1998).

[17] K. P. O’Donnell, R. W. Martin, and P. G. Middleton, Phys. Rev. Lett. 82, 237 (1999).

[18] Y.-H. Cho, T. J. Schmidt, S. Bidnyk, G. H. Gainer, J. J. Song, S. Keller, U. K. Mishra, and S. P. DenBaars, Phys. Rev. B 61, 7571 (2000).

[19] P. R. Kent and A. Zunger, Appl. Phys. Lett. 79, 1997 (2001).

[20] Y. Narukawa, Y. Kawakami, S. Fujita, and S. Nakamura, Phys. Rev. B 59, 10283 (1999).

[21] F. Bernardini and V. Fiorentini, Phys. Rev. B 58, 15292 (1998).

[22] P. Lefebvre, A. Morel, M. Gallart, T. Taliercio, J. Allègre, B. Gil, H. Mathieu, B. Damilano, N. Grandjean, and J. Massies, Appl. Phys. Lett. 78, 1252 (2001).

[23] W. W. Chow, H. Amano, T. Takeuchi, and J. Han, Appl. Phys. Lett. 75, 244 (1999).

[24] Y.-S. Lin, K.-J. Ma, Y.-Y. Chung, S.-W. Feng, Y.-C. Cheng, E.-C. Lin, C. C. Yang, C.-T. Kuo, and J.-S Tsang, J. Cryst. Growth 252, 107 (2003). 